\title{
Automatic Segmentation of Phalanx and Epiphyseal/Metaphyseal Region by Gamma Parameter Enhancement Algorithm
}

\author{
C. W. Hsieh ${ }^{1}$, C. Y. Chen ${ }^{2}$, T. L. Jong ${ }^{3}$, T. C. Liu $^{4}$, C. H. Chiu ${ }^{3}$ \\ ${ }^{1}$ Department of Electrical Engineering, National Chiayi University, No. 300 Syuefu Rd., Chiayi City 60004, Taiwan \\ 2 Instrument Technology Research Center, National Applied Research Laboratories, 20 R\&D Road VI, \\ Hsinchu Science Park, Hsinchu 30076, Taiwan, cychen@itrc.narl.org.tw \\ ${ }^{3}$ Department of Electrical Engineering, National Tsing Hwa University, 101, Section 2, Kuang-Fu Road, \\ Hsinchu 30013, Taiwan \\ ${ }^{4}$ Department of Radiology, Taipei Veterans General Hospital, 201, Section 2, Shih-Pai Road, Taipei 112, Taiwan
}

\begin{abstract}
The performance of bone age assessment is highly correlated with the extraction of bony tissue from soft tissues, and the key problem is how to successfully separate epiphyseal/metaphyseal region of interests (EMROIs) from the background and soft tissue. In our experiment, a series of image preprocessing procedures are used to exclude the background and locate the EMROIs of lefthand radiographs. Subsequently, automatic gamma parameter enhancement is applied to test the two segmentation methods (adaptive two-means clustering algorithm and gradient vector flow snake) among children of different age (the age from 2 to 16 years for 80 girls and boys). Four error measurements of misclassification error, relative foreground area error, modified Hausdorff distances, and edge mismatch, are included to evaluate the segmentation performance. The result shows that the two segmentation algorithms are corresponding to different ranges of optimal gamma parameters. Furthermore, the margin of EMROIs can be obtained more precisely by developing an automatic bone age assessment method with the gamma parameter enhancement.
\end{abstract}

Keywords: Bone age, Epiphyseal/metaphyseal, Segmentation, Gamma parameter enhancement, Adaptive two-means clustering

\section{INTRODUCTION}

$\prod$ HE BONE age (BA) is a significant indication for evaluating the growth potential of children in pediatric endocrinology [1]. Usually, pediatricians or radiologists diagnose the ossifications of carpals, phalanges, ulna and radius, and the epiphysis/metaphysis for a child by determining if the discrepancy between BA and the chronological age (CA) is consistent. Thus, the bone age assessment (BAA) is widely used in clinical practice to examine growth disorders, growth hormone deficiency, and the efficacy of growth therapy in children.

The Greulich and Pyle atlas (GP) [2] and TannerWhitehouse III (TW) method [3] are two extensively used methods that analyze radiographs of the left hand and wrist for determining BA. The GP method is a visual comparison with a standard atlas of hand radiograph, and a pattern can be selected according to how closely it matches the standard. However, the GP method is easily influenced by human subjectivity and qualitative assessment of the ossification status. In comparison, the TW method serves to assess BA according to the detailed shape analyses of several bones of interest and assigning corresponding scores. Subsequently, the sum of all the TW scores is used to compare against the corresponding bone stages. The TW method provides a more objective examination than the GP method, but the drawbacks of complexity and timeconsuming nature result in a seldom use in clinical practice $[4,5]$.

Recently, an increasing attention has been paid to the development of automatic computerized analyses of BA by using a series of image processing techniques. The advantages of BAA include reproducibility and reliability; therefore, many reports focusing on the precise computerbased BAA have been made for improving the performance [6-10]. The critical point of making BAA is how to extract useful characteristics such as bone size and shape. These factors can then be employed to construct a growth model [6]. So, many image processing techniques, such as contrast adjustment, noise filtering, target searching and location combing with the different segmentations including threshold, scanning method, active shape models, are combined to capture the bone region for performing an efficient and accurate analysis [11-19].

Based on the study of $\alpha$-gamma equalization enhancement combing with adaptive two-means clustering algorithm and gradient vector flow (GVF) snake [16], the authors have shown the increased stable performance for the segmentation of epiphyseal/metaphyseal region of interests (EMROIs). However, the enhancement algorithm is using fixed parameters for the EMROIs of distal, middle, and proximal phalanges which are exhibiting too varied values for different backgrounds. To solve the problem, our paper presents an automatic approach of gamma-parameter enhancement to improve performance for the segmentation of EMROIs under good radiographs of hand contrast between bony and soft tissues.

In our study, the EMROIs are extracted by using a series of image preprocessing procedures. Next, the initial image enhancement based on gray level distribution is adopted to make the phalanx contour region clear. Following, the two segmentation methods, the adaptive two-means clustering algorithm and GVF snake, are applied to separate epiphysis 
and metaphysis from the soft tissue. Besides, the experiment makes the statistics to show the segmentation performances to different parameters of gamma selection rules accompanying the two segmentation methods.

\section{SuBJeCt \& METHODS}

To extract left-hand radiographs accurately, a series of image preprocessing procedures are applied to acquire phalangeal bone region of interests (PROIs) and EMROIs. At first, the left hand of the original radiographs is cropped from original radiographs, and then background and unwanted region are excluded. Next, the middle fingertip can be used as the reference to rotate the image in an upward direction. Finally, several image processing strategies are used to extract PROIs and segment the nine EMROIs including the proximal, middle, and distal phalanx of index finger, middle finger, and ring finger, respectively. In Fig.1, the extraction procedures are shown as follows: computing the standard deviation of pixel $I_{L S D}(x, y)$ :

$$
I_{L S D}(x, y)=\frac{1}{5} \sqrt{\sum_{p=x-2}^{x+2} \sum_{q=y-2}^{y+2}\left(I_{p q}-\mu_{x y}\right)^{2}}
$$

where $\mu_{x y}$ is the mean intensity value within the mask.

3. Convolve the $I_{L S D}$ image with a median filter of $7 \times 7$ pixels and apply a threshold process by its mean value to approach a rough hand region, $I_{M E D}$ (see Fig.2c).

4. Use a threshold for the original radiograph by threemeans clustering algorithm in the minimum centroid to obtain $I_{3-\text { means }}$ (see Fig.2d).

5. Combine two images of Fig.2c and Fig.2d with gray color to produce Fig.2e, and the output is defined as

\section{PROI extraction}

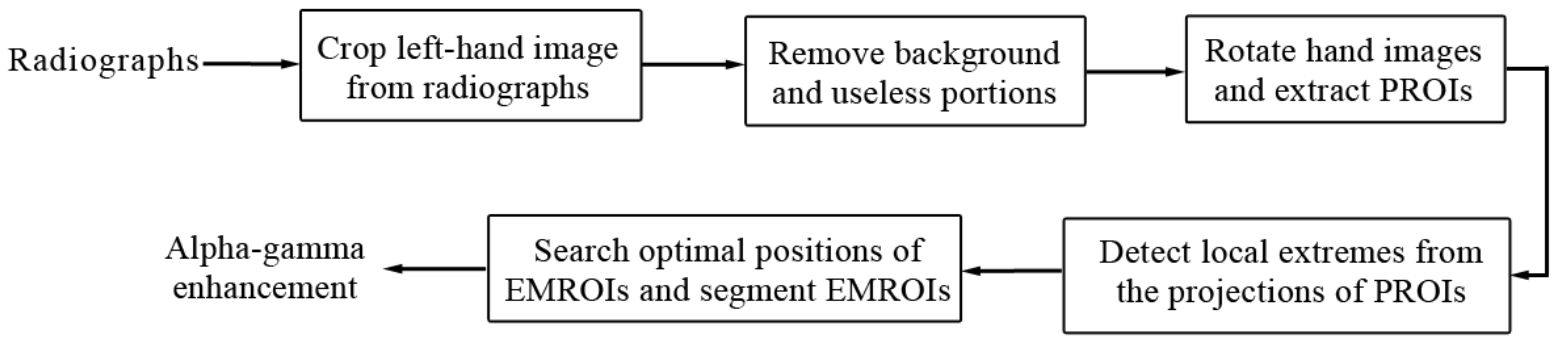

EMROI extraction

Fig.1. The framework for extracting PROIs/EMROIs in hand radiographs.

\subsection{EXTRACTION OF PROIS/EMROIS.}

Below, several image processing techniques are introduced to frame the PROI. Before that, two hands should be separated to obtain the left hand only.

\subsubsection{CROP THE LEFT-HAND REGION.}

The input radiograph usually contains two hands, and the left and right hand may connect to each other in some cases. It is therefore difficult to localize and segment the PROI and EMROI. The technology of cropping the left hand can be accomplished by the previous study [10].

\subsubsection{REMOVE BACKGROUND AND ARM REGIONS,}

In general, $\mathrm{x}$-ray irradiation produces radiographs of nonuniform background, so a scheme of removing background was applied to avoid the segmentation being influenced by regional noise. All details are performed in 9 steps:

1. Acquire the cropped left-hand radiograph, $I$, and resample it by a factor of one-fourth to shorten the execution time (see Fig.2a).

2. Employ the local standard deviations (LSD) with a mask of $5 \times 5$ pixels which was determined empirically (see Fig.2b). The pixel centered at $(x, y)$ is used for

$$
I_{\text {combination }}=I_{M E D}+0.5 \times I_{3-\text { means }}
$$

6. Find out the center of foreground of Fig.2c and apply the 8 neighbor region growing algorithm, with 8 seeds along the lines of eight angles (e.g., $30^{\circ}, 60^{\circ}, 120^{\circ}$, $150^{\circ}, 210^{\circ}, 240^{\circ}, 300^{\circ}$, and $330^{\circ}$, respectively, see Fig.2d) radiating from the center, to separate the foreground from the background image (see Fig.2g).

The obtained image is marked as $I_{r g}$.

7. Do the erosion and dilation for $I_{r g}$ to remove existing noise (see Fig.2h, the $I_{h}$ ).

8. Scan the hand region of Fig.2h line by line from the center to the bottom of the hand, and choose the narrowest width as a cut-off line to remove the arm region, $I_{h_{-} \text {crop }}$ (see Fig.2i).

9. Map Fig.2i onto the original radiogram cropped by the cut-off line to $I_{\text {crop }}$ (see Fig.2j) as shown in:

$$
I_{\text {final }}=\left\{\begin{array}{cc}
I_{\text {crop }}, & I_{h_{-} \text {crop }}>0 \\
0, & I_{h_{-} \text {crop }}=0
\end{array}\right.
$$




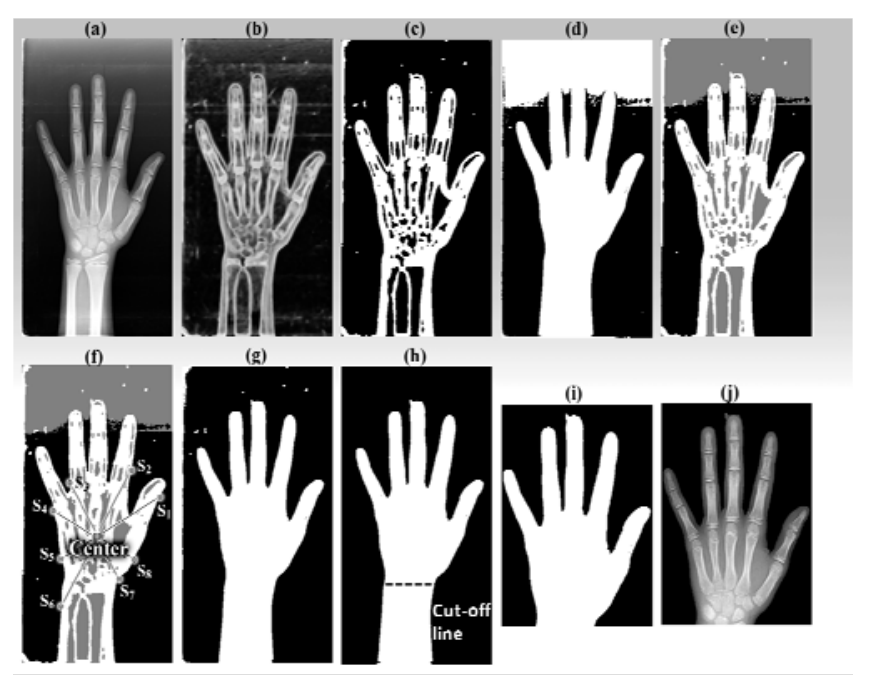

Fig.2. The segmentation procedures of the hand region. (a) Original cropped left-hand radiograph. (b) Local standard deviation image of (a). (c) Threshold image of (b). (d) Threshold image of (a) by three-means algorithm. (e) Combine the (c) and (d) into one. (f) Search foreground image by region growing algorithm. (g) Use the ground image to subtract the estimated background image. (h) Apply erosion and dilation for the image. (i) Remove the arm region from the hand region. (j) Map (i) into original radiograph.

\subsubsection{ROTATE AND CALIBRATE PROIS.}

The following steps are indicative of alignment for the middle fingers to locate PROIs.

1. Input the hand region of Fig.2j.

2. Take the coordinates of the center of the left hand as a reference point, and perform a scanning scheme by computing the radial distances going from the reference point to the PROI boundary with the angle of $30^{\circ}$ to $150^{\circ}$ counterclockwise.

3. Convolve a row mask, [ $\left.\begin{array}{lllll}1 & 1 & 0 & -1 & -1\end{array}\right]$, to search out the three peaks, including index finger, middle finger, and ring finger.

4. Use the maximum peak to rotate the image until the tip of the middle finger almost points upwards to the vertical.

5. Next, place white windows according to the points with local maximum value from the tips of the three fingers to the reference center point (see Fig.3).

6. Use the top window and the bottom window as the reference line, and repeat step 4 for the ring finger and index finger individually.

\subsection{DETECTION AND EXTRACTION OF PROIS.}

The physiological features of EMROIs reveal that the PROIs include three parts and each part has one joint. Therefore, EMROIs can be located by searching out the local extremes from the projection profile of the PROI. The proposed procedures are listed below:

1. Extract each individual PROI and project the intensity into one-dimension vector along the axis from top to bottom.

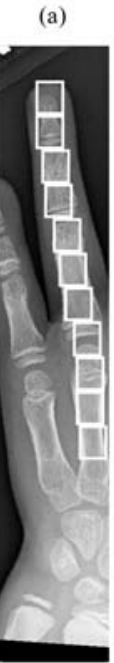

(b)

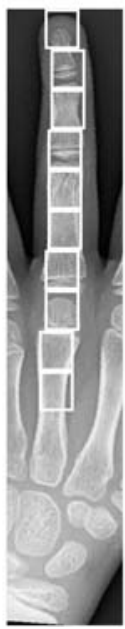

(c)

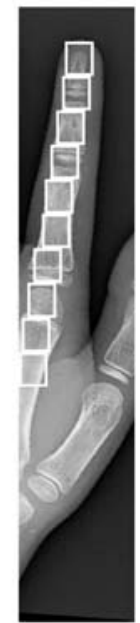

Fig.3. The alignment operation for three fingers from Fig.2j.

(a) The ring finger. (b) The middle finger. (c) The index finger.

2. Convolve the one-dimension vector, $\left[\begin{array}{lllll}1 & 1 & 0 & -1 & -1\end{array}\right]$, to retrieve the gradient information from the different intensity distribution of the PROI. Fig.4a is an example for demonstrating the convolved result.

3. Trace the convolved result and assign a value of 1 for an increasing trend and 0 for the decreasing trend (see Fig.4b). Then, map Fig.4b to Fig.4a to locate the local extremes (see Fig.4c).

4. Take another example in Fig.5. A given ratio of the two lengths between distal and middle phalange, and between middle and proximal phalange, is about threefifth due to the phalange physiology, so we can use the relation to locate three local EMROIs.

5. Finally, three masks $(99 \times 99$ pixels) are retrieved and placed to the three local extremes as the distal, middle, and proximal phalange (see Fig.5b).

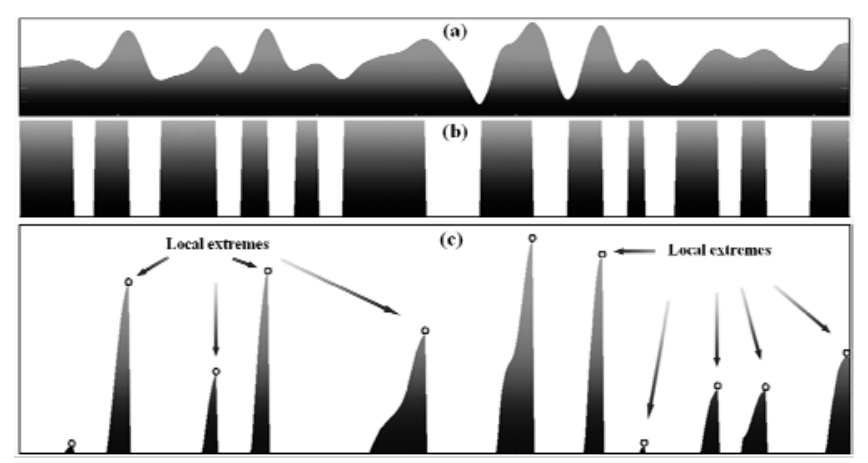

Fig.4. The detection of the local extremes. (a) The projection convolved with the one-dimension vector. (b) Label the increasing and decreasing trends as one and zero, respectively. (c) Map (b) to (a), and locate the positions of local extremes.

\subsection{SEGMENTATION STEPS.}

Although the EMROI is circumscribed within a rectangular window, unfortunately the skeleton and the soft tissue are still hard to identify. Accordingly, we have proposed two segmentation algorithms based on adaptive 


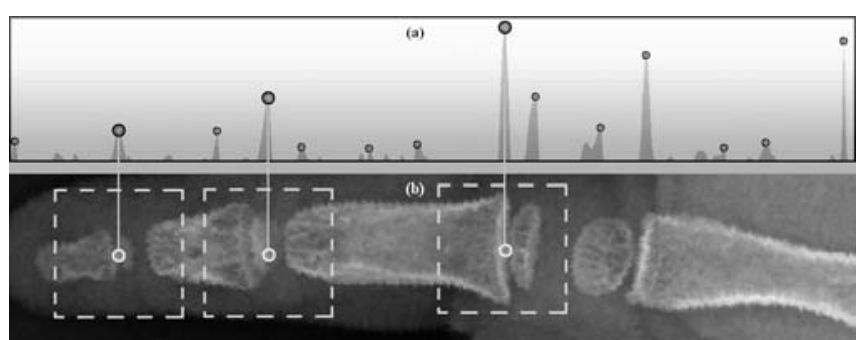

Fig.5. Extraction of EMROIs. (a) The searched local extremes and (b) the framed EMROIs for distal, middle, and proximal phalange, from left to right.

two-means clustering algorithm [17] and GVF snake to combine with gamma parameter enhancement automatically. Before using the operation, an enhancement algorithm is suggested to adjust the gray-level of an image with different effects. The procedures are explained below.

\subsubsection{ADJUSTMENT FOR THE IMAGE WITH GRAY-LEVEL.}

The adjustment for the image gray-level can be defined as:

\subsubsection{SELECTION OF GAMMA VALUES.}

The enhancement can highly influence the segmented images with the changing gamma values, and therefore it is important to choose the appropriate gamma values for the enhancement. This framework is shown in Fig.6. For examining the gamma parameters, the range is defined from 0.1 to 1.5 , and each calculation is added by 0.2 . Then the abovementioned upper/lower bound are used to achieve the gamma parameter enhancement. In the following, all pixels with the gray level values inside the range between lower bound and upper bound should be considered in the enhancement processing.

To evaluate the optimal segmentation of enhanced images, four error measurements of misclassification error (ME), relative foreground area error (RFAE), modified Hausdorff distances (MHD), and edge mismatch (EMM), are used to determine the performance of segmentation [20-22]. The modified version was presented by M. Sezgin and B. Sankur who have adjusted these measures so that their scores vary from 0 (wholly fit to the standard region) to 1 (wholly not fit

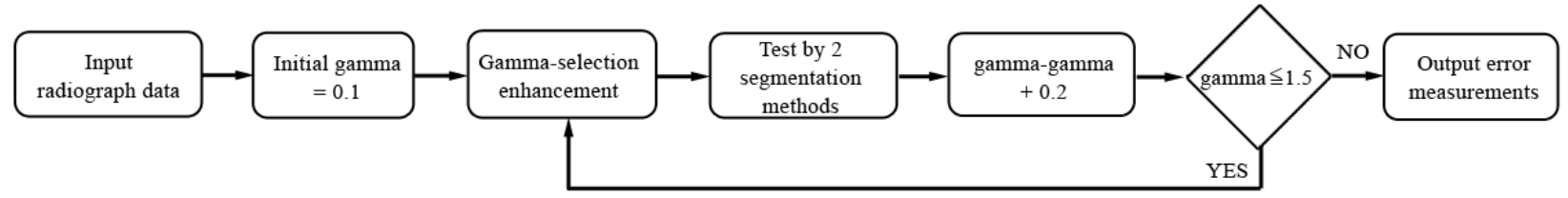

Fig.6. A framework of generating corresponding error measurements.

$$
p_{\gamma}(x, y)=\left\{\begin{array}{cc}
0 & \text { if } g(x, y) \leq L \\
255 & \text { if } g(x, y)>U \\
{\left[\frac{g(x, y)-L}{U-L}\right]^{\gamma}} & \text { otherwise }
\end{array}\right.
$$

$p_{\gamma}(x, y)$ is indicating to adjust the original image $g(x, y)$ for each pixel at the coordinate $(x, y)$. All pixels with gray levels in the range between lower bound (L) and upper bound (U) can be varied with different gamma values and this calculation is used to enhance the image contrast. The details are described below.

\subsubsection{CALCULATION OF UPPER/LOWER BOUND.}

The upper/lower bound is determined by using k-means clustering algorithm. The algorithm classifies each pixel to a predefined cluster which is randomly chosen as a cluster center, namely centroid. The distances between each pixel and all centroids are measured, and the pixel is then assigned to the cluster center with shortest distance. If this assignment is finished, a new centroid is calculated for each cluster with the pixels in it. Once these $\mathrm{k}$ new centroids remain unchanged, the assignment process is regarded as over. Because of the variation for each image, we choose the clustering centers as the upper bound and the lower bound automatically. to the standard region) [22]. The ground-truth image for each case implies the exact region which was defined by an experienced radiologist. Finally, we can output four error measurements with gamma parameter $(\gamma)$ versus CA. Furthermore, the segmentations of each EMROI are recorded as the relationship of CA and 4 error measurements for the adaptive two-mean clustering algorithm and for GVF snake.

\section{RESULTS}

The database, which consists of 3015 radiographs (1531 girls and 1484 boys), was provided by the Department of Radiology, Veterans General Hospital Taipei, and the hand radiographs were scanned in digitized form with the size of $2100 \times 1670$ pixel. The distribution is shown in Fig.7. The testing database contains 160 cases, randomly selected from a group of 2 to 16 year old children with an interval of 2 years, and each age group, for both the girls and the boys, has 10 cases. The other unselected cases were considered as the validating database. In this study, the extracted EMROI images of the testing database were employed to examine the enhancement performances for the gamma selection rule. Then, the derived optimal gamma values can be applied to evaluate the validating database for their segmentation performance, and also the four error measurementss are presented. 


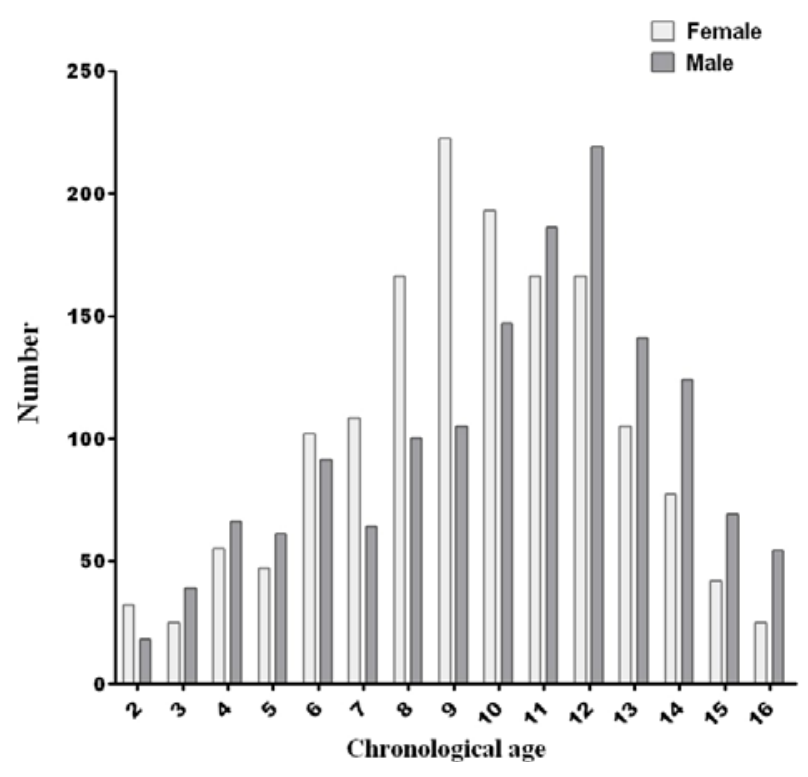

Fig.7. Distributions of radiographs in the database

\subsection{SELECTION RULE OF GAMMA VALUES.}

Based on our proposed selection rules, the optimal gamma parameters can be obtained. Initially, four error measurements were set in the range from 0.1 to 1.5 . As each error measurement is executed with different gamma parameters for the same age group and EMROIs, such as the distal phalange of middle finger, only the performance of error measurement showing the value under $25 \%$ can be accepted. The next step is to assess the intersection of the accepted results for the four error measurements for each gamma parameter into one result. First, if the results of four error measurements all have the accepted results, the corresponding gamma parameter is prior considered as the selected gamma parameter (a label of white circle). Second, if the abovementioned assumption does not apply, and the results of any three error measurements have the accepted results, the corresponding gamma parameter is also chosen as a white circle. The tolerance is defined as that any two error measurements have the accepted results. Once any one or none of the error measurements are in agreement, the gamma parameter belongs to the null set (unaccepted result).

\subsection{EVALUATING GAMMA PARAMETER ENHANCEMENT.}

In the execution process, the gamma parameter enhancement is automatically processed to the remaining EMROIs for girls and boys. Both of the performances show the middle finger as example in a significant range between 0.2 and 0.9 for adaptive two-means clustering algorithm in Table 1(a), and the range between 0.8 and 1.5 for GVF snake, respectively.

Furthermore, Fig.8 demonstrates the differences by adaptive two-means clustering algorithm for showing various patterns with the gamma parameters at $0.5,1$, and 1.5 , respectively . Besides, we also compare the differences for the GVF snake with/without using the gamma of 1.5 in Fig.9. Clearly, the best fit of gamma parameters for the two segmentation algorithms presents the best performances.
Girls’ middle finger

\begin{tabular}{|c|c|c|c|c|c|c|c|c|}
\hline & $\begin{array}{c}0.2 \\
(1,2,3)\end{array}$ & $\begin{array}{c}0.3 \\
(1,2,3)\end{array}$ & $\begin{array}{c}0.4 \\
(1,2,3)\end{array}$ & $\begin{array}{c}0.5 \\
(1,2,3)\end{array}$ & $\begin{array}{c}0.6 \\
(1,2,3)\end{array}$ & $\begin{array}{c}0.7 \\
(1,2,3)\end{array}$ & $\begin{array}{c}0.8 \\
(1,2,3)\end{array}$ & $\begin{array}{c}0.9 \\
(1,2,3)\end{array}$ \\
\hline 2 & & & $\bullet$ & ০০০ & $\bigcirc \bigcirc$ & $\bigcirc \bullet$ & & \\
\hline 4 & $\bullet \bullet \bullet$ & $\bullet \bullet \bullet$ & $\bullet \bullet \bullet$ & ০০০ & $০ \bullet$ & $\bigcirc \bullet$ & $\bullet$ & 0 \\
\hline 6 & $\bullet \bullet \bullet$ & $\bullet \bullet \bullet$ & $\bullet$ & $\bigcirc \bullet$ & $\bigcirc \bullet$ & ০৫ & $\bullet \bullet \bullet$ & $\bullet \bullet \bullet$ \\
\hline 8 & $\bullet \bullet \bullet$ & $\bullet \bullet$ & $\bullet$ & ০৫ & ○८ & ○॰ & $\bullet \bullet \bullet$ & D०० \\
\hline 10 & $\bullet \bullet$ & $\bullet$ & ০৫ & ०৫ & ○•• & $\bullet \bullet \bullet$ & $\bullet \bullet \bullet$ & $\bullet \bullet \bullet$ \\
\hline 12 & $\bullet \bullet \bullet$ & $\bullet \bullet \bullet$ & 000 & $\bigcirc \bullet$ & ○০ & $\bullet \bullet \bullet$ & $\bullet \bullet \bullet$ & $\bullet \bullet \bullet$ \\
\hline 14 & $\bullet \bullet \bullet$ & $\bullet$ & ০০০ & 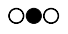 & $\bullet \bullet$ & ○८ & $\bullet \bullet \bullet$ & $\bullet \bullet \bullet$ \\
\hline 16 & $\bullet \bullet \bullet$ & $\bullet \bullet \bullet$ & ০০০ & O০০ & ○০ & & $\bullet \bullet \bullet$ & $\bullet \bullet \bullet$ \\
\hline
\end{tabular}

Boys' middle finger

\begin{tabular}{|c|c|c|c|c|c|c|c|c|}
\hline & $\begin{array}{c}0.2 \\
(1,2,3)\end{array}$ & $\begin{array}{c}0.3 \\
(1,2,3)\end{array}$ & $\begin{array}{c}0.4 \\
(1,2,3)\end{array}$ & $\begin{array}{c}0.5 \\
(1,2,3)\end{array}$ & $\begin{array}{c}0.6 \\
(1,2,3)\end{array}$ & $\begin{array}{c}0.7 \\
(1,2,3)\end{array}$ & $\begin{array}{c}0.8 \\
(1,2,3)\end{array}$ & $\begin{array}{c}0.9 \\
(1,2,3)\end{array}$ \\
\hline 2 & & & ০০০ & $\bullet$ & $\bigcirc$ & & & \\
\hline 4 & $\bullet$ & $\bullet \bullet \bullet$ & ০০০ & ০০০ & ০০ & & 100 & 0 \\
\hline 6 & $\bullet \bullet \bullet$ & $\bullet \bullet \bullet$ & ০০০ & ০০০ & ০০ & $\bullet \bullet \bullet$ & $\bullet \bullet \bullet$ & $\bullet \bullet \bullet$ \\
\hline 8 & $\bullet$ & $\bullet$ & ০০০ & ০৫ & ○८ & $\bullet \bullet \bullet$ & $\bullet \bullet \bullet$ & $\bullet \bullet \bullet$ \\
\hline 10 & $\bullet \bullet \bullet$ & $\bullet \circ ০$ & ০০০ & ০০ & ০৫ & $\bullet \bullet \bullet$ & $\bullet \bullet \bullet$ & $\bullet \bullet \bullet$ \\
\hline 12 & ०८ & 00 & 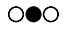 & $\bullet \bullet$ & $\bullet \bullet \bullet$ & $\bullet \bullet \bullet$ & $\bullet \bullet \bullet$ & $\bullet \bullet \bullet$ \\
\hline 14 & $\bullet \bullet \bullet$ & ০০ & ০০০ & ০০০ & $\bullet \bullet$ & $\bullet \bullet \bullet$ & $\bullet \bullet \bullet$ & $\bullet \bullet \bullet$ \\
\hline 16 & $\bullet \bullet \bullet$ & $\bullet$ & ০০০ & ০০ & 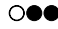 & $\bullet \bullet \bullet$ & $\bullet \bullet \bullet$ & $\bullet \bullet$ \\
\hline
\end{tabular}

Table 1. Result of gamma parameters versus CA is executed by adaptive two-means clustering algorithm for girls and boys, and the white circles and black circles represent the accepted results and unaccepted result, respectively (distal, medial and proximal phalange are marked by the number of 1,2 and 3 ).

\section{Girls' middle finger}

\begin{tabular}{|c|c|c|c|c|c|c|c|c|}
\hline & $\begin{array}{c}0.8 \\
(1,2,3)\end{array}$ & $\begin{array}{c}0.9 \\
(1,2,3)\end{array}$ & $\begin{array}{c}1 \\
(1,2,3)\end{array}$ & $\begin{array}{c}1.1 \\
(1,2,3)\end{array}$ & $\begin{array}{c}1.2 \\
(1,2,3)\end{array}$ & $\begin{array}{c}1.3 \\
(1,2,3)\end{array}$ & $\begin{array}{c}1.4 \\
(1,2,3)\end{array}$ & $\begin{array}{c}1.5 \\
(1,2,3)\end{array}$ \\
\hline 2 & $\bullet \bullet \bullet$ & $\bullet \bullet \bullet$ & $\bullet \bullet \bullet$ & $\bullet \bullet \bullet$ & $\bullet \bullet \bullet$ & ০০০ & ০৫ & ০০০ \\
\hline 4 & $\bullet \bullet \bullet$ & $\bullet \bullet \bullet$ & $\bullet \bullet \bullet$ & $\bullet \bullet \bullet$ & $\bullet \bullet \bullet$ & 000 & ০০০ & 000 \\
\hline 6 & $\bullet \bullet \bullet$ & $\bullet \bullet \bullet$ & $\bullet \bullet$ & $\bullet \bullet$ & $\bullet \bullet$ & 000 & ০০ & 000 \\
\hline 8 & $\bullet \bullet \bullet$ & $\bullet \bullet \bullet$ & $\bullet \bullet \bullet$ & $\bullet \bullet \bullet$ & $\bullet \bullet \bullet$ & O०০ & O०० & 000 \\
\hline 10 & $\bullet \bullet \bullet$ & $\bullet \bullet$ & $\bullet \bullet$ & $\bullet \bullet \bullet$ & $\bullet \bullet \bullet$ & $\bullet$ & $\bullet$ & ০০ \\
\hline 12 & $\bullet \bullet \bullet$ & $\bullet \bullet \bullet$ & $\bullet \bullet$ & $\bullet \bullet \bullet$ & $\bullet \bullet \bullet$ & ০০৫ & ০০৫ & ০০ \\
\hline 14 & & & $\bullet \bullet \bullet$ & & & ০০৫ & ০০৫ & 000 \\
\hline 16 & & & & & & 000 & 000 & ০০০ \\
\hline
\end{tabular}

Boys' middle finger

\begin{tabular}{|c|c|c|c|c|c|c|c|c|}
\hline & $\begin{array}{c}0.8 \\
(1,2,3)\end{array}$ & $\begin{array}{c}0.9 \\
(1,2,3)\end{array}$ & $\begin{array}{c}1 \\
(1,2,3)\end{array}$ & $\begin{array}{c}1.1 \\
(1,2,3)\end{array}$ & $\begin{array}{c}1.2 \\
(1,2,3)\end{array}$ & $\begin{array}{c}1.3 \\
(1,2,3)\end{array}$ & $\begin{array}{c}1.4 \\
(1,2,3)\end{array}$ & $\begin{array}{c}1.5 \\
(1,2,3)\end{array}$ \\
\hline 2 & $\bullet \bullet \bullet$ & $\bullet \bullet \bullet$ & $\bullet \bullet \bullet$ & & & ০০ & ০০ & O০০ \\
\hline 4 & $\bullet \bullet \bullet$ & & & & $\bullet \bullet$ & 000 & 000 & 000 \\
\hline 6 & $\bullet \bullet \bullet$ & 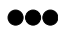 & & & & ০০০ & 000 & 000 \\
\hline 8 & $\bullet \bullet \bullet$ & $\bullet \bullet \bullet$ & $\bullet \bullet \bullet$ & • & $\bullet \bullet \bullet$ & ০৫ & ०৫ & $\bigcirc \bullet$ \\
\hline 10 & $\bullet \bullet \bullet$ & $\bullet \bullet \bullet$ & $\bullet \bullet \bullet$ & $\bullet \bullet \bullet$ & $\bullet \bullet \bullet$ & ০০০ & 000 & ০০ \\
\hline 12 & $\bullet \bullet \bullet$ & $\bullet \bullet \bullet$ & $\bullet \bullet \bullet$ & $\bullet \bullet \bullet$ & $\bullet \bullet \bullet$ & ০০০ & $\bullet$ & ০০০ \\
\hline 14 & $\bullet \bullet \bullet$ & ८०८ & $\bullet \bullet \bullet$ & $\bullet \bullet \bullet$ & $\bullet \bullet \bullet$ & O०০ & 000 & 000 \\
\hline 16 & & $\bullet \bullet \bullet$ & $\bullet \bullet \bullet$ & $\bullet \bullet \bullet$ & $\bullet \bullet \bullet$ & ০০ & ০০ & ০০ \\
\hline
\end{tabular}

Table 2. Result of gamma parameters versus CA is executed by GVF snake for girls and boys, and the white circles and black circles represent the accepted results and unaccepted result, respectively. (distal, medial and proximal phalange are marked by the number of 1,2 and 3) 


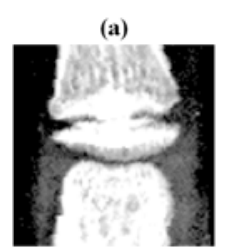

(d)
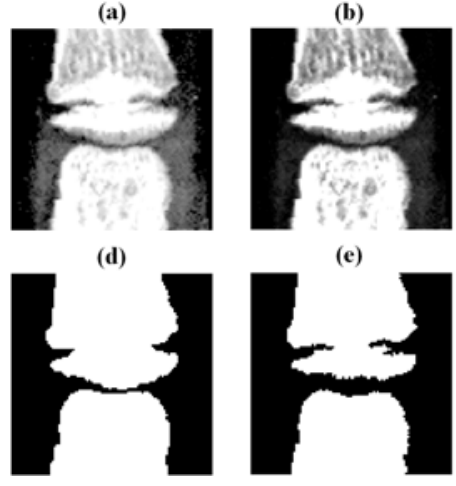

(e)

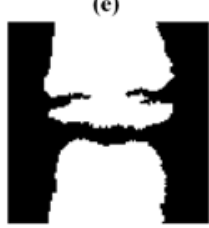

Fig.8. An example of adaptive two-means segmentation with gamma parameter enhancement for (a) (c) with gamma parameters at $0.5,1$, and 1.5 and their corresponding segmented images in (d) (f). (a)

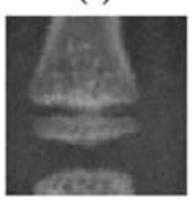

(c)

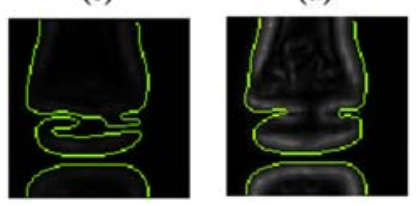

Fig.9. An example of segmentation result by GVF snake. (a) Original image. (b) Enhancement of original image with gamma parameter at 1.5. (c) Segmented image by GVF snake for (a). (d) Segmented image by GVF snake for (b).

\subsection{EVALUATION FOR THE VALIDATING DATABASE.}

To evaluate the exact performance of the proposed gamma parameter enhancement algorithm, the abovementioned four error measurements are taken for validating the proposed algorithm. Because the best-fit situation can be observed with smaller error values for the adaptive two-means clustering algorithm in the gamma of 0.5 , and for the GVF snake in the gamma of 1.5, both gamma parameters are chosen for the following calculations. In examining the validating database, the four error measurements of 9 segments of the phalanges (including proximal, middle, distal phalange of index finger, middle finger, and ring finger) for one case are averaged to show how the proposed methods improve the segmentation. The comparisons of the two segmentation algorithms show the statistics of mean and standard deviation in Fig.10a for girls and Fig.10b for boys. The results explain that our proposed gamma parameter enhancement has better improvement for the segmentation.

\section{Discussion/CONCLUSIONS}

In this study, we present a series of image preprocessing procedures for the segmentation and locating of PROIs /EMROIs from radiographs to overcome the complex tasks. An accurate and efficient segmentation is critical for feature
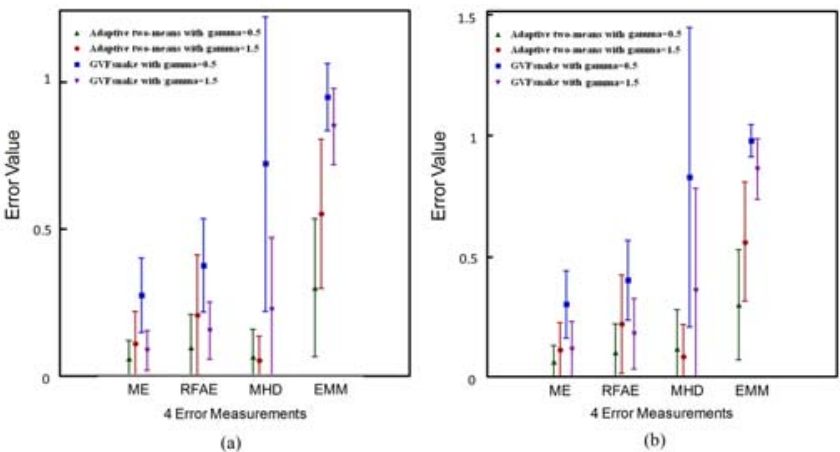

Fig.10. Descriptive statistics of the mean and the standard deviation in the comparison between two segmentation methods for (a) girls and (b) boys.

extraction of BAA, so many previous studies were devoted to the development of different kinds of segmentation methods. Usually, the existence of spatial and intensity relationships between pair pixels can have great influences for the segmentation, especially for the soft tissue in the EMROI which can be recognized as the specific target of bony tissue and vice versa. Such situation can cause a poor contrast for bony tissue to the soft tissue. So, a gamma parameter enhancement algorithm was proposed to improve the image contrast and preserve edges of bony tissue excluding the encircling soft tissue.

With the automatic computation of upper/lower bound, our findings demonstrate the enhancement algorithm by using various gamma parameters, revealing the nonlinear relationship between CA and gamma parameters. The statistic results presented in Fig.8 and Fig.9 showing how the appropriate gamma parameters can help achieve the best approach, and the specified gamma parameters, 0.5 for adaptive two-means clustering algorithm and 1.5 for GVF snake, can be derived from the testing database. Also, in Fig.10a and Fig.10b for the validation database, the optimal results appear for the specified gamma parameters for the two segmentation methods with the smaller mean and standard deviation.

The observation demonstrates that the segmentation has better results with gamma parameter enhancement than with fixed gamma parameters for the two segmentation methods. Furthermore, such great variations of gamma parameters between two segmentations may be caused by their initial conditions. For example, initial segmentation of the adaptive two-means clustering algorithm should be obtained to exclude the background of EMROIs first, so that the processing considers merely the related contrast of bony and soft tissues. Therefore, gamma parameters using adaptive two-means clustering algorithm are not proper for higher values. In another case of GVF snake, the initial contour generated by deformable model can be sustained far away from the object boundary. Thus, the contrast must be deservedly conspicuous for the GVF snake to segment EMROIs.

The performed experimental results confirm usability and feasibility of both the applied extraction and locating of 
PROIs/EMROIs based on the gamma parameter enhancement for the adaptive two-means clustering algorithm and GVF snake. Avoiding the segmentation error, our contribution can be of assistance to BAA and monitoring of growth therapies. In the future, we plan to build up an automatic BAA for advanced work.

\section{ACKNOWLEDGMENT}

This work was supported by the National Science Council R.O.C. under Grant NSC 100-2221-E-007-119 and NSC 100-2218-E-492-027.

\section{REFERENCES}

[1] Poznanski, A.K. (1984). The Hand in Radiologic Diagnosis: With Gamuts and Pattern Profiles. Philadelphia: Saunders.

[2] Greulich, W.W., Pyle, S.I. (1971). Radiographic Atlas of Skeletal Development of the Hand and Wrist, Second Edition. Stanford University Press.

[3] Tanner, J.M., Healy, M.J., Goldstein, H., Cameron, N. (2001). Assessment of Skeletal Maturity and Prediction of Adult Height: TW3 Method. Philadelphia: Saunders.

[4] Gertych, A., Zhang, A., Sayre, J., PospiechKurkowska, S., Huang, H.K. (2007). Bone age assessment of children using a digital hand atlas. Comput. Med. Imaging Graph., 31 (4-5), 322-331.

[5] Zhang, A., Gertych, A., Liu, B.J. (2007). Automatic bone age assessment for young children from newborn to 7-year-old using carpal bones. Comput. Med. Imaging Graph., 31 (4-5), 299-310.

[6] Aja-Fernández, S., de Luis-García, R., MartínFernández, M.Á., Alberola-López, C. (2004). A computational TW3 classifier for skeletal maturity assessment. A computing with words approach. $J$. Biomed. Inform., 37 (2), 99-107.

[7] Hsieh, C.W., Liu, T.C., Jong, T.L., Tiu, C.M. (2010). A fuzzy-based growth model with principle component analysis selection for carpal bone-age assessment. Med. Biol. Eng. Comput., 48 (6), 579-588.

[8] Thodberg, H.H., Kreiborg, S., Juul, A., Pedersen, K.D. (2009). The BoneXpert method for automated determination of skeletal maturity. IEEE Trans. Med. Imaging, 28 (1), 52-66.

[9] Tristan-Vega, A., Arribas, J.I. (2008). A radius and ulna TW3 bone age assessment system. IEEE Trans. Biomed. Eng., 55 (5), 1463-1476.
[10] Hsieh, C.W., Jong, T.L., Tiu, C.M. (2007). Bone age estimation based on phalanx information with fuzzy constrain of carpals. Med. Biol. Eng. Comput., 45 (3), 283-295.

[11] Hsieh, C.W., Liu, T.C., Jong, T.L., Chen, C.Y., Tiu, C.M., Chan, D.Y. (2011). Fast and fully automatic phalanx segmentation using a grayscale-histogram morphology algorithm. Opt. Eng., 50 (8), 087007.

[12] Long-Ke, R., Ling, H., Zhong, C. (2012). A location and extraction method research for phalange ROI and carpal ROI from left hand radiography. Advanced Materials Research, 340, 259-265.

[13] Giordano, D., Spampinato, C., Scarciofalo, G., Leonardi, R. (2011). An automatic system for skeletal bone age measurement by robust processing of carpal and epiphysial/metaphysial bones. IEEE Trans. Instrum. Meas., 59 (10), 2539-2553.

[14] BoneXpert System, http://www.bonexpert.com/index.php

[15] Xu, C., Prince, J.L. (1998). Snakes, shapes, and gradient vector flow. IEEE Trans. Image Processing, 7 (3), 359-369.

[16] Lin, H.H., Shu, S.G., Kuo, S.W., Wang, C.H., Chan, Y.P., Yu, S.S. (2009). Alpha-gamma equalizationenhanced hand radiographic image segmentation scheme. Opt. Eng., 48 (10), 107001.

[17] Yong, Y., Chongxun, Z., Pan, L. (2004). A novel fuzzy c-means clustering algorithm for image thresholding. Meas. Sci. Rev., 4, 11-19.

[18] Brodic, D. (2011). Methodology for the evaluation of the algorithms for text line segmentation based on extended binary classification. Meas. Sci. Rev., 11 (3), 11-19.

[19] Yasnoff, W.A., Mui, J.K., Bacus, J.W. (1977). Error measures for scene segmentation. Patt. Recog., 9 (4), 217-231.

[20] Kampke, T., Kober, R. (1998). Nonparametric optimal binarization. In $14^{\text {th }}$ International Conference on Pattern Recognition. IEEE, Vol. 1, 27-29.

[21] Dubuisson, M.P., Jain, A.K. (1994). A modified Hausdorff distance for object matching. In Proceedings of the $12^{\text {th }}$ IAPR International Conference on Pattern Recognition. IEEE, Vol. 1, 566-568.

[22] Sezgin, M., Sankur, B. (2004). Survey over image thresholding techniques and quantitative performance evaluation. J. Electron. Imaging, 13 (1), 146-168.

Received November 24, 2011. Accepted February 28, 2012. 